\title{
TDP-43 Proteinopathies:
}

Neurodegenerative Protein Misfolding
Diseases without Amyloidosis

\author{
Linda K. Kwong $^{a}$ Kunihiro Uryu ${ }^{a}$ John Q. Trojanowski ${ }^{a}$ b Virginia M.-Y. Lee ${ }^{a, b}$ \\ ${ }^{a}$ Center for Neurodegenerative Disease Research, Department of Pathology and Laboratory Medicine, and \\ ${ }^{b}$ Institute on Aging, University of Pennsylvania School of Medicine, Philadelphia, Pa., USA
}

\section{Key Words}

TDP-43 - Frontotemporal lobar degeneration •

Amyotrophic lateral sclerosis • Neurodegenerative disease

Frontotemporal dementia

\begin{abstract}
In this review, we summarize recent advances in understanding frontotemporal lobar degeneration (FTLD), amyotrophic lateral sclerosis (ALS) and related neurodegenerative disorders that are collectively known as TDP-43 proteinopathies, since transactive response DNA-binding protein 43 (TDP-43) was recently shown to be the major component of the ubiquitinated inclusions that are their pathological hallmarks. TDP-43 proteinopathies are distinct from most other neurodegenerative disorders because TDP-43 inclusions are not amyloid deposits. Besides TDP-43-positive inclusions, both sporadic and familial forms of FTLD and ALS have the pathologic TDP-43 signature of abnormal hyperphosphorylation, ubiquitination and C-terminal fragments in affected brain and spinal cord, suggesting that they share a common mechanism of pathogenesis. Thus, these findings support the concept that FTLD and ALS represent a clinicopathologic spectrum of one disease, that is, TDP-43 proteinopathy.
\end{abstract}

Copyright $\odot 2008$ S. Karger AG, Basel

\section{Introduction}

Frontotemporal lobar degeneration (FTLD) refers to a clinically, genetically and pathologically heterogeneous group of neurodegenerative disorders and is the second most common form of dementia in those under the age of 65, after Alzheimer's disease (AD), with prevalence estimates ranging between 3.3 and 25.4 per 100,000 [1, 2]. Clinically, FTLD is characterized by progressive changes in social, behavioral and/or language dysfunction. Current research criteria divide FTLD into 3 clinical syndromes: frontotemporal dementia (FTD), primary progressive nonfluent aphasia (PNFA) and semantic dementia (SD) [3]. FTD, the most common clinical form, primarily presents with personality and behavioral changes often associated with disinhibition, apathy and lack of insight, while PNFA and SD present predominantly with language dysfunctions characterized by effortful speech and phonemic errors, as well as severe problems with naming objects and understanding words, respectively. In the early phase of these syndromes, there is relative preservation of memory, but, in addition, patients may display movement abnormalities, such as parkinsonism or motor neuron disease (MND) [4-6].

The term 'frontotemporal lobar degeneration' reflects the prominent frontal and temporal lobe atrophy associated with neuronal loss and gliosis on neuropathological

Virginia M.-Y. Lee, PhD, Center for Neurodegenerative Disease Research Department of Pathology and Laboratory Medicine

University of Pennsylvania School of Medicine, HUP, Maloney 3rd Floor 36th and Spruce Streets, Philadelphia, PA 19104-4283 (USA)

Tel. +1 215662 6399, Fax +1 215349 5909, E-Mail vmylee@mail.med.upenn.edu 
examination. In addition, as in most adult-onset neurodegenerative diseases, abnormal proteinaceous inclusions are present in neurons and glial cells. Thus, based on immunohistochemistry (IHC), FTLD can be broadly divided into 2 pathological categories: (1) those cases with tau-positive inclusions known as tauopathies, such as FTLD with Pick bodies, corticobasal degeneration, progressive supranuclear palsy, argyrophilic grain disease and neurofibrillary tangle-only dementia, and (2) those cases without tau pathology. The latter can be further categorized by the presence or absence of ubiquitinpositive, tau- and $\alpha$-synuclein-negative inclusions (UBIs) [4]. Those with UBIs are termed FTLD-U, which is the most common neuropathological subtype of FTLD, and together with FTLD tauopathies they account for $>95 \%$ of FTLDs [7-10].

Despite exhaustive efforts, using biochemical techniques similar to those used in studying other neurodegenerative diseases, such as tauopathies and synucleinopathies, and the recent identification of genetic defects underlying familial forms of FTLD-U, such as mutations in the progranulin gene $(G R N)[11,12]$ and valosin-containing gene (VCP) [13], the protein-building blocks of UBIs in FTLD-U remained unknown until recently when we identified transactive response (TAR) DNA-binding protein 43 (TDP-43) as the major disease protein in UBIs in both FTLD-U and amyotrophic lateral sclerosis (ALS) [14]. Hence, this review will focus on the identification and pathobiology of TDP-43 as the disease protein in sporadic and familial FTLD-U with and without MND as well as in sporadic and familial ALS. In addition, our current knowledge on TDP-43 neuropathology in other neurodegenerative diseases will also be described. Finally, the implications of these findings for the diagnosis and treatment of this new class of disorders, which collectively can be referred to as neurodegenerative TDP-43 proteinopathies, will be discussed.

\section{Identification of Ubiquitinated, Phosphorylated and Truncated TDP-43 as the Disease Protein in FTLD-U}

Our initial approach to identify the disease protein in FTLD-U was to adopt various biochemical enrichment techniques similar to those used for other neurodegenerative diseases, such as the isolation of tau containing paired helical filaments in $\mathrm{AD}$ and the isolation of $\alpha$ synuclein in Parkinson's disease (PD) [15]. The rationale for this approach is the recognition that most neurodegenerative diseases share a common pathogenetic mech- anism characterized by accumulation of intracellular and extracellular misfolded protein aggregates in the central nervous system. The underlying disease mechanism involves the misfolding of normally soluble proteins into $\beta$-pleated conformation and the deposition of the misfolded proteins into insoluble fibrillar aggregates with the physical and chemical properties of amyloid. For example, most of these neurodegenerative disease-specific aggregates (as exemplified by tangles and plaques in $\mathrm{AD}$ and Lewy bodies, LBs, in PD) are filamentous and display the ultrastructural and dye-binding properties of amyloid; that is, the formation of $\sim 10$-nm-wide fibrils with $\beta$-pleated sheet structures that stain with silver, Congo red, thioflavin S or other amyloid dyes. However, UBIs in FTLD-U are not detected by amyloid-binding dyes, suggesting that FTLD-U might be a unique neurodegenerative proteinopathy characterized by protein misfolding in the absence of brain amyloidosis. Thus, it was no surprise that biochemical techniques used to purify filamentous aggregates, such as the methods developed to isolate paired helical filaments in $\mathrm{AD}$, were unsuccessful in iso-

Fig. 1. Scheme of a combined biochemical and immunologic approach used to identify TDP-43 as the disease protein in FTLD-U and ALS. a This panel outlines the steps used in the approach. b Urea-soluble protein extracts of cortical gray matter from normal control and FTLD-U separated by SDS-PAGE and immunoblotted with anti-ubiquitin. The box indicates high-molecularmass $(\mathrm{Mr}>250 \mathrm{kDa})$ smear of proteins from FTLD-U extracts used for immunization of mice (c) for monoclonal antibody $(\mathrm{mAb})$ production. $\mathbf{d}$ IHC results from the screen of hybridoma supernatants for immunodetection of UBIs by new mAbs. Note the characteristic TDP-43-positive cytoplasmic inclusions in the hippocampal dentate granule cells in an FTLD-U brain and the clearance of physiological nuclear TDP-43 in cells with inclusions (arrows). e Immunoblot analysis of urea-soluble protein extracts from control and FTLD-U cortical gray matter with novel mAbs demonstrates the unique protein profile or banding pattern in the FTLD-U compared to the control brain (molecular mass markers are shown on the left). ${ }^{*}$ Unique $\sim 25-\mathrm{kD}$ a band in FTLD-U.f This two-dimensional SDS-PAGE shows representative urea-soluble protein extracts from FTLD-U brains immunoblotted with novel mAbs (the pI range from 3 to 10 is shown on the bottom, while molecular mass markers are shown on the left). Liquid chromatographic mass spectrometry analysis was performed on Coomassie blue-stained sister gels, identifying TDP-43 as the disease protein. $\mathbf{g}$ TDP-43 immunoblot analysis of urea-soluble protein extracts from control and FTLD-U brains are shown here and this demonstrates the disease-specific biochemical profile of TDP-43 with additional $\sim 25$-kDa bands $\left({ }^{*}\right)$, a $\sim 45-\mathrm{kDa}$ band $\left({ }^{* *}\right)$ above the normal $45-\mathrm{kDa}$ band and the high-molecular-weight protein smear $\left({ }^{* *}\right)$ in extracts from FTLD-U brains, which are not detectable in control brain extracts (molecular mass markers are shown on the left). 


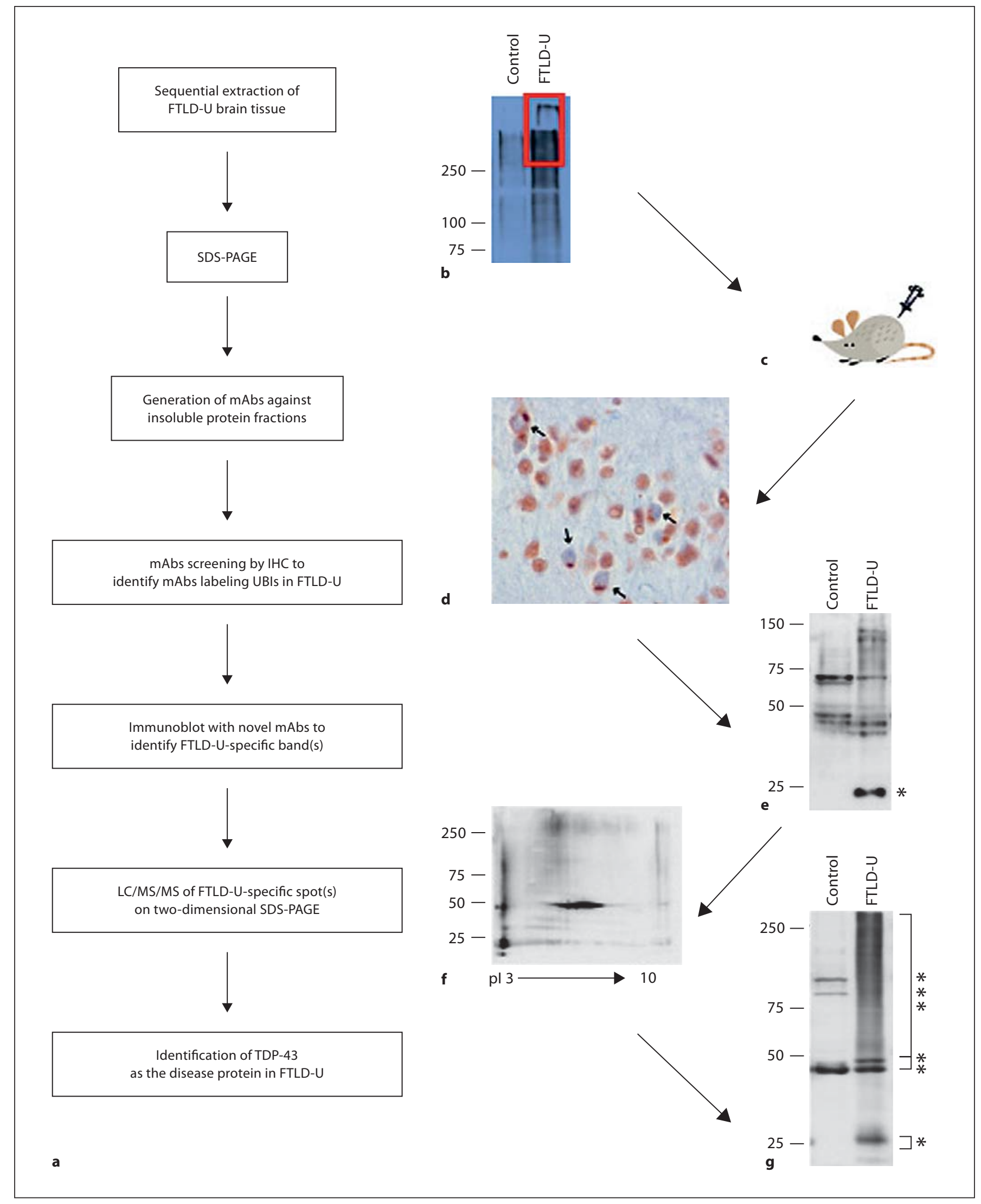

Neurodegenerative TDP-43

Neurosignals 2008;16:41-51 
lating UBIs from FTLD-U brains. Furthermore, the task of finding the protein components of UBIs in FTLD-U was limited by: (1) the heterogeneous morphology and distribution of the UBIs in different brain regions among different FTLD-U cases; (2) the relatively low abundance of the UBIs; and (3) the lack of reagents other than antiubiquitin antibodies for the detection of UBIs. Thus, although multiple biochemical techniques including fractionation of disease brains to enrich for UBIs and twodimensional differential gel electrophoresis proteomic approaches were used to identify the disease protein or proteins in FTLD-U, they were uniformly uninformative. For these reasons, we pursued an alternative strategy combining biochemical and immunologic approaches [16]. To do this, we made the assumption that the increased levels of ubiquitin-immunoreactive, high-molecular-weight smears in insoluble material from biochemical fractionation of FTLD-U brains after sodium dodecyl sulfate polyacrylamide gel electrophoresis (SDS-PAGE), compared to normal brain insoluble material, contain the disease protein in UBIs (fig. 1b). Thus, we used the highmolecular-mass $(\mathrm{Mr}>250 \mathrm{kDa})$ protein smear from SDSPAGE of FTLD-U for immunizing mice to generate potentially useful antibody probes for our FTLD-U studies (fig. 1c). However, this approach was complicated by the fact that the distribution of UBIs in FTDL-U brains is heterogeneous and their abundance is low relative to tangles and plaques in AD brain. Based on the morphology and laminar distribution of ubiquitin-positive neuronal cytoplasmic inclusions (NCIs), neuronal intranuclear inclusions (NIIs) and dystropohic neurites (DNs) in affected cortical brain regions, at least 3 pathological subtypes of FTLD-U were distinguished [16]. Briefly, subtype 1 is characterized by a predominance of long neuritic profiles over NCIs in superficial cortical layers, subtype 2 shows a predominance of NCIs in both superficial and deep cortical layers, and in subtype 3 ring-shaped NCIs as well as short neuritic and intranuclear profiles are present predominantly in the superficial cortical layers (fig. 2). This system of FTLD-U subtyping was independently confirmed by others [17].

Thus, to take into account the possibility of multiple disease protein(s) among the different FTLD-U subtypes, we immunized mice with high-molecular-mass $(\mathrm{Mr}>250$ $\mathrm{kDa}$ ) insoluble material obtained from brains of each of the 3 FTLD-U subtypes to general antibodies recognizing UBIs from each of these subtypes. Over 50,000 hybridomas were generated, and they were all screened by IHC using brain sections from all 3 FTLD-U subtypes (fig. 1d). We were successful in generating novel mono- clonal antibodies (mAbs) specific for subtypes 1 and 2, but not 3. Interestingly, subtype-specific mAbs immunolabeled all UBIs in the corresponding FTLD-U subtype used to generate the mAbs, but not UBIs in other subtypes [16]. Importantly, a few of these mAbs were able to distinguish FTLD-U subtypes and control brain extracts in immunoblots. For example, the subtype-specific $m A b s$ labeled a unique protein band in the respective subtype extracts and not in other subtypes or control brains (fig. 1e). Thus, using these novel mAbs, we performed extensive protein analysis of extracts from FTLD-U brains, including two-dimensional SDS-PAGE and liquid chromatographic mass spectrometry, and we were able to determine the identity of the subtype-specific bands (fig. 1f). Interestingly, both subtype 1- and 2-specific bands were identified as N-terminally truncated species of TDP-43, which suggests that the $\mathrm{mAbs}$ recognize subtype-specific conformations or posttranslational modifications of a C-terminal breakdown and/or cleavage product of TDP43 (fig. 1e).

TDP-43 is a 414-amino-acid nuclear protein, encoded by the TARDBP gene on chromosome 1 . The gene was initially cloned from a genomic screen for cellular factors that bind to the TAR DNA of HIV type 1 [18] and was also identified independently as part of a complex involved in the splicing of the cystic fibrosis transmembrane conductance regulator gene $[19,20]$. More recently, it was shown to regulate the expression of the mouse SP10 gene [21] and the splicing of intron and exon of the apoA-II gene [22]. TDP-43 is a highly conserved protein that is ubiquitously expressed in all tissues examined, including heart, lung, liver, spleen, kidney, muscle and brain [19]. It contains 2 RNA recognition motifs as well as a glycine-rich C-terminal sequence [19,23], which may be required for its exon skipping and splicing inhibitory activity $[24,25]$. This is consistent with the finding that the C-terminal domain of TDP-43 binds to several proteins of the heterogeneous nuclear ribonucleoprotein family, which are involved with the biogenesis of mRNA $[24,26]$. Finally, TDP-43 may also act as scaffold for nuclear bodies through interaction with survival motor neuron protein [27].

Despite the pathological heterogeneity among the FTLD-U subtypes and the specificity of the novel mAbs for specific subtypes, IHC using commercially available TDP-43 antibodies showed TDP-43 to be the major component in the characteristic inclusions, NCIs, NIIs and DNs, in all 3 FTDL-U subtypes (fig. 2). This was further supported by the colocalization of ubiquitin and TDP43-positive inclusions in double-labeling fluorescence 


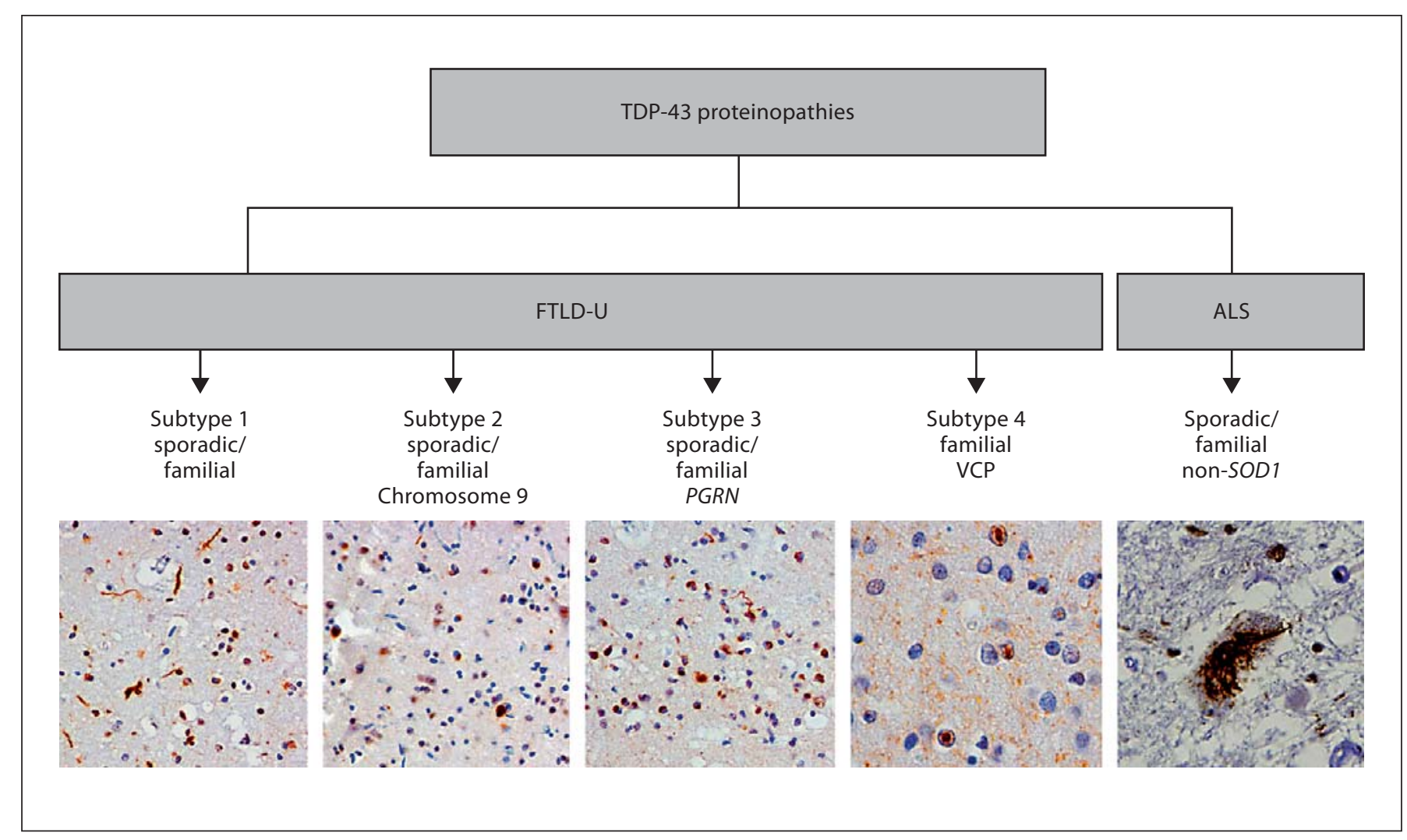

Fig. 2. Classification of TDP-43 proteinopathies. Accumulation of TDP-43-positive inclusions defines a new class of proteinopathies, including FTLD-U and ALS, that are distinct from all other neurodegenerative protein misfolding diseases because, unlike most of these other disorders, TDP-43 proteinopathies are not central nervous system amyloidoses. As described in the text here, based on the TDP-43 IHC profile, 4 histological subtypes of FTLD-U can be delineated; note that some, but not all, are associated with different familial forms of FTLD-U. Images below each subtype and ALS illustrate the characteristic histological features by anti-TDP-43 IHC.

studies [14]. Notably, the characteristic inclusions of other neurodegenerative disorders, including those that are signature lesions of Pick's disease, corticobasal degeneration, progressive supranuclear palsy, FTD with parkinsonism linked to chromosome 17 (FTDP-17) caused by tau gene (MAPT) mutations, neuronal intermediate filament inclusion disease, basophilic inclusion body disease, hereditary diffuse leukoencephalopathy with spheroids, AD, argyrophilic grain disease, neurofibrillary tangle-only dementia, PD, dementia with LBs, multiple system atrophy and trinucleotide repeat diseases were virtually negative for TDP-43 [14, 28]. Thus, these important negative findings reinforced the initial observations that TDP-43 is a highly specific marker for FTLD-U [14]. Moreover, our finding that TDP-43 is the major disease protein in FTLD-U has been confirmed by many other laboratories [28-31].

Neurodegenerative TDP-43

Proteinopathies

\section{TDP-43 Pathology in Sporadic and Familial FTLD-U with and without MND}

It is now clear that virtually all different morphological forms of UBIs in FTLD-U with and without MND, including ubiquitin-positive NCIs, NIIs and DNs, are TDP-43 positive $[14,28,30]$. The robust TDP-43 immunostaining in affected cortical regions of FTLD-U has the same distinct morphology and distribution pattern as those detected with anti-ubiquitin antibodies, suggesting that anti-TDP-43 antibodies detect the same neuropathology in subtypes $1-3$ as antibodies to ubiquitin $[14,28$, 30]. Furthermore, UBIs in the motor neurons of spinal cord and brain stem in FTLD-U with and without clinical signs of MND were immunopositive for TDP-43 [14, 28]. When present, DNs were most numerous in neocortical areas of the frontal and temporal lobes; rarely, nu-

Neurosignals 2008;16:41-51 45 
merous DNs were seen in the CA1 subfield of the hippocampus. TDP-43-positive NCIs and NIIs were variably distributed throughout cortical areas and the hippocampus, including the dentate gyrus $[14,28,30]$. Notably, physiological TDP-43 is detectable in the nuclei of unaffected neurons, but it is dramatically absent in the nuclei of neurons with NCIs, suggesting that TDP- 43 redistributes from nucleus to cytoplasm in affected neurons (fig. 1d) $[14,28,30]$. This redistribution may have significant consequences on the physiological and pathobiological functions of TDP-43. In addition, TDP-43 IHC revealed previously unrecognized white matter pathology in FTLD-U cases, comprised of TDP-43-positive glial cytoplasmic inclusions and rare thread-like inclusions that are negative for ubiquitin, tau and $\alpha$-synuclein [31]. Based on their morphology and double-labeling experiments, these affected glial cells most likely are oligodendrocytes. TDP-43-positive glial inclusions can be found throughout the frontal and temporal lobes in a subset of FTLD-U cases with the highest density in subtype 2 and 3 cases, providing further evidence for pathological heterogeneity in FTLD-U [31]. Moreover, scattered glial inclusions can be found in brainstem and spinal cord regions in FTLD-U cases with motor neuron involvement. This suggests that white matter pathology might also contribute to the neurodegenerative process and the cognitive and motor impairments seen in patients affected by FTLD-U.

Subtype 1 is characterized by an abundance of long neuritic profiles predominantly in the superficial cortical laminae, with few or no NCIs and NIIs (fig. 2). There are also TDP-43-positive inclusions in the hippocampal dentate granule cells. Interestingly, all the inclusions can be labeled with subtype 1-specific mAbs, but not with subtype 2-specific mAbs. Glial pathology is rare [31]. Subtype 1 cases tend to be sporadic and without MND, but there are a few cases with MND and/or family history that have subtype 1 pathology [28]. However, no specific genetic defect has been associated with subtype 1 . This subtype is the most common in patients with $\mathrm{SD}[17$, 32].

The predominant inclusions in subtype 2 cases are NCIs mainly in the upper cortical laminae, but also throughout the entire cortical thickness (fig. 2). There are also TDP-43-positive inclusions in the hippocampus. These inclusions are positive with subtype $2 \mathrm{mAbs}$, but not subtype $1 \mathrm{mAbs}$. In addition, numerous TDP-43-positive granular 'pre-inclusions' are present in the neurons of affected regions, but they are not ubiquitin positive [28]. In some subtype 2 cases, TDP-43-positive inclu- sions, similar to UBIs found in ALS cases, are detected in motor neurons in the hypoglossal nuclei and ventral horn of the spinal cord and this could explain why many subtype 2 patients often present with clinical signs of MND [17]. Moreover, subtype 2 is associated frequently with abundant glial pathology in affected cortical, brainstem and spinal cord regions [31]. Most interestingly, among familial FTLD cases, only those with a confirmed linkage to a locus on chromosome 9, but not those with a GRN or $V C P$ mutation, are subtype 2 cases [28]. Finally, the morphology and distribution of TDP-43 pathology in FTLDMND are also consistent with subtype 2 [32].

Subtype 3 cases are characterized by an abundance of small neuritic profiles and NCIs, often ring shaped, predominantly in the superficial cortical layers (fig. 2). Furthermore, lentiform NIIs can be found in affected cortical regions. In addition, glial pathology is often present in affected cortical regions. These findings have been confirmed and extended by others [30,33], and none of the inclusions in subtype 3 cases stain with subtype 1 or $2 \mathrm{mAbs}$. The morphology and distribution of TDP-43 pathology in $G R N(+)$ cases are indicative of subtype 3 [14, $28,33]$. Interestingly, there are clinical parkinsonism features in many of the GRN(+) cases [34]. Furthermore, PNFA cases also typically show subtype 3 TDP-43 pathology [32].

Mutations in a gene known as $V C P$ on chromosome 9 , that encodes the valosin-containing protein (VCP), also have been shown to cause FTD with inclusion body myositis and Paget's disease of bone [35]. However, TDP-43 pathology in $V C P(+)$ cases does not correlate with subtypes 1-3. A unique neuropathology of FTLD-U with robust TDP-43 and ubiquitin staining of DNs and NIIs with few NCIs in the affected cortical regions and no detectable inclusions in the hippocampal dentate granule cells was evident (fig. 2) [13, 36]. The DNs and NIIs are most abundant in the upper cortical layers [36]. Thus, $V C P(+)$ cases can be classified as FTLD-U subtype 4 [28]. So far, this histological subtype has not been described in sporadic or other familial FTLD-U cases.

Finally, not all FTLD-U cases have TDP-43-positive UBIs. In our large multicenter study of FTLD-U, UBIs from 7/192 cases were TDP-43 negative [28]. Of these, 2 cases were sporadic, 1 familial with unidentified gene linkage and 4 with mutations in the charged multivesicular body protein $2 \mathrm{~B}$ gene (CHMP2B). CHMP $2 \mathrm{~B}$ on chromosome 3 was recently linked to FTD in a large Danish pedigree [37]. Although few cases have been examined post-mortem and the neuropathology was originally described as 'dementia lacking distinctive histopathology', 
recent studies have revealed ubiquitin-positive NCIs in the dentate fascia and sparse inclusions in the neocortex $[28,38]$; however, these inclusions are TDP-43 negative.

Although there is a predictable relationship between pathological TDP-43 subtypes and clinical phenotypes $[17,32]$, the significance of TDP-43 subtypes in the formation of cytoplasmic, neuritic and nuclear inclusions is unknown. The identification of 2 different mutant genes (PGRN, VCP) and another unidentified gene on chromosome 9 that are linked to FTLD-U with TDP-43 pathology should provide important clues for the elucidation of pathogenic pathways that lead to the accumulation of pathological TDP-43. Indeed, it is tempting to speculate that there might be a distinct TDP-43 pathological pathway unique to each TDP-43 subtype.

\section{Biochemical Studies of TDP-43 in FTLD-U}

Biochemical analyses of TDP-43 in affected brain regions of FTLD-U and control cases revealed that TDP-43 is pathologically modified in FTLD-U [14]. These modifications include the presence of a disease-specific hyperphosphorylated protein band that migrates at $\sim 45 \mathrm{kDa}$, ubiquitinated high-molecular-mass smears, and C-terminal fragments at $\sim 25 \mathrm{kDa}$ are evident in the Sarkosylinsoluble, urea-soluble protein extracts of FTLD-U brains (fig. 1g). Although the solubility and expression levels of the physiological TDP- 43 species at $43 \mathrm{kDa}$ did not appear to differ between FTLD-U and control brains, the signature of pathological TDP-43 was detected in Sarkosyl-insoluble urea extracts of gray and white matters from affected regions of all familial and sporadic FTLD$\mathrm{U}$ brains with TDP- 43 inclusions, albeit to a variable extent $[14,28,31,36]$. This variation seems to reflect the density of TDP-43-positive inclusions detected by IHC [36].

Among neurodegenerative diseases with aggregates containing misfolded proteins, mutations are often found in the gene encoding the misfolded protein in familial forms of the diseases. For example, mutations in the microtubule-associated protein tau gene (MAPT) are pathogenic for FTDP-17, the other major familial forms of FTD wherein tau tangles are the major neuropathology. Thus, it is not surprising that searching for mutations or disease-associated variations in the TARDBP gene is a priority for several laboratories. So far, no pathogenic mutations in TARDBP have been identified in familial FTLD and there is no evidence that variations in the TARDBP gene increase risk for sporadic FTLD [39-41]. This raises the possibility that the redistribution and abnormal accumulation of TDP- 43 might be a consequence of different underlying disease processes rather than the cause of the disease. However, since TDP-43 is a highly conserved protein, pathogenic variations in the TARDBP gene might be very rare, or they may not have the potent effects of autosomal dominant gene mutations like those in MAPT, but continued efforts are needed to determine if changes in TARDBP result in familial forms of FTLD-U.

\section{TDP-43 in ALS}

ALS is a common neurodegenerative MND characterized by the relentless loss of both upper motor neurons (UMNs) and lower motor neurons (LMNs), resulting in progressive weakness, muscular wasting and spasticity leading to paralysis and death within 3-5 years [42]. The etiology is unknown and there is currently no effective treatment. Most ALS cases are sporadic (sALS), but about $10 \%$ of ALS patients have a positive family history (fALS), of which $\sim 20 \%$ result from missense mutations in the $\mathrm{Cu} / \mathrm{Zn}$ superoxide dismutase 1 gene (SOD1) [42, 43]. Clinically, about $20 \%$ of ALS cases also develop or present with FTD [44], but $~ 50 \%$ of ALS patients will develop evidence of cognitive impairment during the course of their illness [45]. Besides the loss of UMNs and LMNs, the hallmark neuropathological findings in ALS include degeneration of corticospinal tracts and abnormal accumulation of insoluble ubiquitin-positive inclusions in the cytoplasm of degenerating motor neurons [46]. These UBIs often appear as either filamentous skeins or compact, LB-like round inclusions [14, 46-48] and these are most common in LMNs. Until recently, little was known about the identity of the protein(s) in these inclusions. The overlapping clinical and neuropathological features between FTLD and ALS prompted us to investigate the role of TDP-43 in ALS. In our initial report, we demonstrated that all UBIs in motor neurons of sALS were robustly double-labeled by TDP-43 and ubiquitin antibodies, but not by subtype 1- and 2-specific mAbs. Likewise, the UBIs present in the hippocampus and the frontal and temporal cortex of some ALS cases [49] were also immunolabeled by TDP-43. Furthermore, results from biochemical analysis of Sarkosyl-insoluble, urea-soluble spinal cord and brain extracts from affected regions of ALS showed the same biochemical signature of pathological TDP-43 as seen in FTLD-U. These initial findings were rapidly confirmed by others $[47,48,50]$. As described in 
a subset of FTLD-U cases, additional oligodendroglial pathology in the affected brain regions is a consistent finding in sALS [47]. Moreover, TDP-43-positive inclusions have been reported in some patients with primary lateral sclerosis, a form of MND characterized by the progressive loss primarily of UMNs [50].

Thus, we participated in a multicenter neuropathological study of TDP-43 in a large series of sALS and fALS cases. Surprisingly, while pathological TDP-43 detected by IHC is a consistent finding in sALS and non-SOD1fALS, no TDP-43-positive inclusions were detected by the same methods in a series of 15 SOD1-fALS cases caused by 7 different SOD1 mutations [47]. Similar findings were reported in 2 Japanese SOD1-fALS cases [48], while a third study on 2 SOD1-fALS cases reported the absence of TDP-43 in hyaline conglomerate inclusions and noted the presence of ubiquitin-negative, TDP-43immunoreactive profiles in the cytoplasm of motor neurons [51]. The remarkable finding of TDP-43 pathology in sALS and non-SOD1-fALS, but not in SOD1-fALS was further confirmed by the presence of disease-specific biochemical TDP-43 signature in the spinal cord and brainstem of sALS and non-SOD1-fALS, but not in cases of fALS caused by SOD1 mutations [47].

Taken together, the results provide histological and biochemical evidence that ALS and FTLD-U represent a clinicopathological spectrum of neurodegenerative disorders characterized by pathological accumulations of abnormal TDP-43 aggregates. The absence of TDP-43 pathology in the vast majority of SOD1-fALS cases implies that motor neuron degeneration in these cases may result from mechanisms that are different from those underlying sALS and non-SOD1-fALS.

\section{TDP-43 Pathology in Other Neurodegenerative Diseases}

As discussed in the previous section, TDP-43 is a highly specific marker for FTLD-U lesions, although rare inclusions in some tauopathies do contain both TDP-43 and tau immunoreactivities $[29,52]$. This specificity of TDP-43 for distinctly different lesions contrasts sharply with ubiquitin which tags many disease proteins for degradation and is present in many neurodegenerative disease-specific lesions. Moreover, the distinctness of TDP43 lesions permits the investigation of TDP-43 pathology in the setting of other neurodegenerative disorders to determine if TDP-43 lesions occur concurrently with other neuropathologies, such as neurofibrillary tangles and
LBs. For example, the presence of tau and $\alpha$-synuclein inclusions in addition to those formed by TDP-43 has been reported recently in members of $2 G R N(+)$ families [53]. Surprisingly, TDP-43 inclusions similar to those found in FTLD-U and ALS have been reported in AD as well as in Guam parkinsonism-dementia complex (GPDC) and Guam ALS (G-ALS). A study of 123 AD cases showed that $\sim 20 \%$ harbored TDP-43 pathology in the hippocampus [54]. A similar finding was noted in our multicenter FTLD-U study, where $\sim 16 \%$ of the AD cases had TDP-43-positive inclusions in the hippocampus [28]. The presence of TDP- 43 pathology in AD was confirmed and further extended to cases of PD, PD with dementia and dementia with LBs [55]. In this study of TDP-43 in LB-related diseases, TDP-43 inclusions were found in the hippocampus of $\sim 29 \%$ of dementia with LBs/AD, $\sim 7 \%$ of PD and $\sim 19 \%$ of PD with dementia cases. G-PDC and G-ALS are neurodegenerative diseases specific to the Chamorro population on Guam. Clinically, they are characterized by either progressive cognitive impairment similar to $\mathrm{AD}$, or motor impairments similar to $\mathrm{PD}$ or MMD. A study of 9 G-PDC brains showed the presence of TDP-43-positive inclusions in the frontotemporal and hippocampal regions as well as the disease-specific abnormal TDP-43 biochemical profile [52]. This finding was independently observed in a study of large series of G-PDC and G-ALS cases [56]. TDP-43 pathology was found to varying degrees in the gray and white matter of neocortical regions as well as in the hippocampus of GPDC. Surprisingly, TDP-43-positive neuronal and glial inclusions were present in the spinal cord not just in GALS but also G-PDC, albeit to a lesser extent than in GALS. This finding in Guam neurodegenerative diseases supports the hypothesis that FTD and ALS may represent the 2 extremes of a continuous clinicopathological spectrum of one disease. Further investigation is needed to determine the significance of concomitant TDP-43 pathology in other neurodegenerative diseases.

\section{Implications of TDP-43 Proteinopathy for the Diagnosis and Treatment of FTDs and ALS}

Our initial discoveries [14] that TDP-43 abnormalities are the underlying neuropathological substrates of familial and sporadic forms of FTLD-U with and without MND as well as ALS have been confirmed and extended by numerous follow-up studies to familial and sporadic forms of FTLD-U with and without MND as well as to sALS and non-SOD1-fALS. It is evident that a new class 
of neurodegenerative disorders, TDP- 43 proteinopathies, has emerged that is defined by distinct TDP-43 pathologies [for review, see 57-59]. This will have significant implications for the diagnosis and treatment of FTDs and ALS. This is abundantly clear based on similar earlier advances in understanding mechanisms of tau- and amyloid $\beta$-mediated neurodegeneration in $\mathrm{AD}$ and other tauopathies as well as $\alpha$-synuclein-mediated neurodegeneration in PD and related synucleinopathies [15]. However, drugs that are designed to ameliorate or reverse brain amyloidosis, such as many in clinical trial now for $\mathrm{AD}$, are unlikely to have efficacy in FTLD-U because TDP- 43 inclusions are formed by TDP- 43 aggregates that do not have the physical and chemical properties of amyloids $[28,54]$. However, efforts to abrogate TDP-43 aggregation could inform efforts to target prefibrillar forms of amyloid such as oligomeric forms of tau, amyloid $\beta$ or $\alpha$-synuclein in other neurodegenerative diseases characterized by brain amyloidosis. Furthermore, the development of assays to monitor the levels of normal and pathological TDP-43 in plasma and/or cerebrospinal fluid as biomarkers of FTLD-U and ALS could lead to the development of diagnostic tests to distinguish TDP-43 proteinopathies from other clinically similar neurodegenerative disorders, such as tauopathies. In addition, the development of imaging ligands that enable the detection of TDP-43 neuropathology in living patients will provide a powerful tool not only for diagnosis, but also for following the response of patients with a neurodegenerative TDP-43 proteinopathy to disease-modifying therapies when they become available. Thus, recognition that TDP43 pathology underlies and links FTLD-U and ALS is a significant driver of efforts to develop more effective therapies for these disorders.

\section{Conclusion}

In summary, the identification of TDP-43 as the major component of the pathologic inclusions in most cases of sporadic and familial FTLD-U with and without MND as well as sALS and non-SOD1-fALS has resolved a longstanding enigma regarding the nature of the ubiquitinated inclusions in these disorders. Pathological TDP-43 is the most sensitive and specific marker for FTLD-U and ALS, and antibodies to TDP-43 allowed the detection of previously unrecognized glial pathology in FTLD-U and ALS. Thus, despite the significant clinical, genetic and neuropathological heterogeneity of FTLD-U and ALS, ubiquitinated, hyperphosphorylated and C-terminal fragments of TDP-43 are present in both neurodegenerative disorders in addition to TDP-43-positive inclusions, thereby implicating TDP-43 in novel and unifying mechanisms of FTLD and ALS pathogenesis. Thus, these observations support the idea that FTLD-U and ALS may represent the 2 extremes of a continuous clinicopathological spectrum of one disease, that is, TDP-43 proteinopathies (fig. 2). The important consequences of the discoveries summarized in this review will reorient efforts to develop better strategies for the diagnosis and treatment of FTDs and ALS.

\section{Acknowledgements}

This work was funded by the National Institutes of Health (AG10124 and AG17586). V.M.-Y.L. is the John H. Ware III Chair of Alzheimer's Research and J.Q.T. is the William Maul MeaseyTruman G. Schnabel, Jr., MD Professor of Geriatric Medicine and Gerontology. The authors would like to thank the families of our patients who made this research possible.

\section{References}

1 Harvey RJ, Skelton-Robinson M, Rossor $\mathrm{MN}$ : The prevalence and causes of dementia in people under the age of 65 years. J Neurol Neurosurg Psychiatry 2003;74:1206-1209.

$\checkmark 2$ Ratnavalli E, Brayne C, Dawson K, Hodges JR: The prevalence of frontotemporal dementia. Neurology 2002;58:1615-1621.

-3 Neary D, Snowden JS, Gustafson L, Passant U, Stuss D, Black S, Freedman M, Kertesz A, Robert PH, Albert M, Boone K, Miller BL, Cummings J, Benson DF: Frontotemporal lobar degeneration: a consensus on clinical diagnostic criteria. Neurology 1998;51: 1546-1554.
-4 Forman MS, Farmer J, Johnson JK, Clark CM, Arnold SE, Coslett HB, Chatterjee A, Hurtig HI, Karlawish JH, Rosen HJ, Van D, V, Lee VM, Miller BL, Trojanowski JQ, Grossman M: Frontotemporal dementia: clinicopathological correlations. Ann Neurol 2006;59:952-962.

5 Kertesz A, Munoz DG: Frontotemporal dementia. Med Clin North Am 2002;86:501518.

6 6 Lomen-Hoerth C, Anderson T, Miller B: The overlap of amyotrophic lateral sclerosis and frontotemporal dementia. Neurology 2002; 59:1077-1079.
7 Hodges JR, Davies RR, Xuereb JH, Casey B, Broe M, Bak TH, Kril JJ, Halliday GM: Clinicopathological correlates in frontotemporal dementia. Ann Neurol 2004;56:399-406.

8 Johnson JK, Diehl J, Mendez MF, Neuhaus J, Shapira JS, Forman M, Chute DJ, Roberson ED, Pace-Savitsky C, Neumann M, Chow TW, Rosen HJ, Forstl H, Kurz A, Miller BL: Frontotemporal lobar degeneration: demographic characteristics of 353 patients. Arch Neurol 2005;62:925-930. 
-9 Lipton AM, White CL III, Bigio EH: Frontotemporal lobar degeneration with motor neuron disease-type inclusions predominates in 76 cases of frontotemporal degeneration. Acta Neuropathol (Berl) 2004;108: 379-385.

-10 Shi J, Shaw CL, Du PD, Richardson AM, Bailey KL, Julien C, Stopford C, Thompson J, Varma A, Craufurd D, Tian J, PickeringBrown S, Neary D, Snowden JS, Mann DM: Histopathological changes underlying frontotemporal lobar degeneration with clinicopathological correlation. Acta Neuropathol (Berl) 2005;110:501-512.

- 11 Baker M, Mackenzie IR, Pickering-Brown SM, Gass J, Rademakers R, Lindholm C, Snowden J, Adamson J, Sadovnick AD, Rollinson S, Cannon A, Dwosh E, Neary D, Melquist S, Richardson A, Dickson D, Berger Z, Eriksen J, Robinson T, Zehr C, Dickey CA, Crook R, McGowan E, Mann D, Boeve B, Feldman H, Hutton M: Mutations in progranulin cause tau-negative frontotemporal dementia linked to chromosome 17. Nature 2006;442:916-919.

12 Cruts M, Gijselinck I, van der ZJ, Engelborghs S, Wils H, Pirici D, Rademakers R, Vandenberghe R, Dermaut B, Martin JJ, van Duijn C, Peeters K, Sciot R, Santens P, De Pooter T, Mattheijssens M, Van den BM, Cuijt I, Vennekens K, De Deyn PP, KumarSingh S, Van Broeckhoven C: Null mutations in progranulin cause ubiquitin-positive frontotemporal dementia linked to chromosome 17q21. Nature 2006;442:920-924.

-13 Forman MS, Mackenzie IR, Cairns NJ, Swanson E, Boyer PJ, Drachman DA, Jhaveri BS, Karlawish JH, Pestronk A, Smith TW, Tu PH, Watts GD, Markesbery WR, Smith CD, Kimonis VE: Novel ubiquitin neuropathology in frontotemporal dementia with valosincontaining protein gene mutations. J Neuropathol Exp Neurol 2006;65:571-581.

- 14 Neumann M, Sampathu DM, Kwong LK, Truax AC, Micsenyi MC, Chou TT, Bruce J, Schuck T, Grossman M, Clark CM, McCluskey LF, Miller BL, Masliah E, Mackenzie IR, Feldman H, Feiden W, Kretzschmar HA, Trojanowski JQ, Lee VM: Ubiquitinated TDP-43 in frontotemporal lobar degeneration and amyotrophic lateral sclerosis. Science 2006;314:130-133.

15 Skovronsky DM, Lee VM, Trojanowski JQ: Neurodegenerative diseases: new concepts of pathogenesis and their therapeutic implications. Annu Rev Pathol Mech Dis 2006;1: 151-170.

16 Sampathu DM, Neumann M, Kwong LK, Chou TT, Micsenyi M, Truax A, Bruce J, Grossman M, Trojanowski JQ, Lee VM: Pathological heterogeneity of frontotemporal lobar degeneration with ubiquitin-positive inclusions delineated by ubiquitin immunohistochemistry and novel monoclonal antibodies. Am J Pathol 2006;169:13431352.
17 Mackenzie IR, Baborie A, Pickering-Brown S, Du PD, Jaros E, Perry RH, Neary D, Snowden JS, Mann DM: Heterogeneity of ubiquitin pathology in frontotemporal lobar degeneration: classification and relation to clinical phenotype. Acta Neuropathol (Berl) 2006;112:539-549.

$18 \mathrm{Ou}$ SH, Wu F, Harrich D, Garcia-Martinez LF, Gaynor RB: Cloning and characterization of a novel cellular protein, TDP-43, that binds to human immunodeficiency virus type 1 TAR DNA sequence motifs. J Virol 1995;69:3584-3596.

19 Buratti E, Dork T, Zuccato E, Pagani F, Romano M, Baralle FE: Nuclear factor TDP-43 and SR proteins promote in vitro and in vivo CFTR exon 9 skipping. EMBO J 2001;20: 1774-1784.

20 Buratti E, Brindisi A, Pagani F, Baralle FE: Nuclear factor TDP-43 binds to the polymorphic TG repeats in CFTR intron 8 and causes skipping of exon 9: a functional link with disease penetrance. Am J Hum Genet 2004;74:1322-1325.

-21 Acharya KK, Govind CK, Shore AN, Stoler $\mathrm{MH}$, Reddi PP: cis-Requirement for the maintenance of round spermatid-specific transcription. Dev Biol 2006;295:781-790.

22 Mercado PA, Ayala YM, Romano M, Buratti E, Baralle FE: Depletion of TDP 43 overrides the need for exonic and intronic splicing enhancers in the human apoA-II gene. Nucleic Acids Res 2005;33:6000-6010.

23 Ayala YM, Pantano S, D’Ambrogio A, Buratti E, Brindisi A, Marchetti C, Romano M, Baralle FE: Human, Drosophila, and C. elegans TDP43: nucleic acid binding properties and splicing regulatory function. J Mol Biol 2005;348:575-588.

24 Buratti E, Brindisi A, Giombi M, Tisminetzky S, Ayala YM, Baralle FE: TDP-43 binds heterogeneous nuclear ribonucleoprotein $\mathrm{A} /$ B through its C-terminal tail: an important region for the inhibition of cystic fibrosis transmembrane conductance regulator exon 9 splicing. J Biol Chem 2005;280:3757237584.

25 Wang HY, Wang IF, Bose J, Shen CK: Structural diversity and functional implications of the eukaryotic TDP gene family. Genomics 2004;83:130-139.

-26 Dreyfuss G, Matunis MJ, Pinol-Roma S, Burd CG: hnRNP proteins and the biogenesis of mRNA. Annu Rev Biochem 1993;62: 289-321.

27 Wang IF, Reddy NM, Shen CK: Higher order arrangement of the eukaryotic nuclear bodies. Proc Natl Acad Sci USA 2002;99:1358313588.
28 Cairns NJ, Neumann M, Bigio EH, Holm IE, Troost D, Hatanpaa KJ, Foong C, White CL, III, Schneider JA, Kretzschmar HA, Carter D, Taylor-Reinwald L, Paulsmeyer K, Strider J, Gitcho M, Goate AM, Morris JC, Mishra M, Kwong LK, Stieber A, Xu Y, Forman MS, Trojanowski JQ, Lee VM, Mackenzie IR: TDP-43 in familial and sporadic frontotemporal lobar degeneration with ubiquitin inclusions. Am J Pathol 2007;171:227-240.

-29 Arai T, Hasegawa M, Akiyama H, Ikeda K, Nonaka T, Mori H, Mann D, Tsuchiya K, Yoshida M, Hashizume Y, Oda T: TDP-43 is a component of ubiquitin-positive tau-negative inclusions in frontotemporal lobar degeneration and amyotrophic lateral sclerosis. Biochem Biophys Res Commun 2006; 351:602-611.

30 Davidson Y, Kelley T, Mackenzie IR, Pickering-Brown S, Du PD, Neary D, Snowden JS, Mann DM: Ubiquitinated pathological lesions in frontotemporal lobar degeneration contain the TAR DNA-binding protein, TDP-43. Acta Neuropathol (Berl) 2007;113: 521-533.

-31 Neumann M, Kwong LK, Truax AC, Vanmassenhove B, Kretzschmar HA, Van Deerlin VM, Clark CM, Grossman M, Miller BL, Trojanowski JQ, Lee VM: TDP-43-positive white matter pathology in frontotemporal lobar degeneration with ubiquitin-positive inclusions. J Neuropathol Exp Neurol 2007; 66:177-183.

32 Snowden J, Neary D, Mann D: Frontotemporal lobar degeneration: clinical and pathological relationships. Acta Neuropathol (Berl) 2007;114:31-38.

-33 Mackenzie IR, Baker M, Pickering-Brown S, Hsiung GY, Lindholm C, Dwosh E, Gass J, Cannon A, Rademakers R, Hutton M, Feldman $\mathrm{HH}$ : The neuropathology of frontotemporal lobar degeneration caused by mutations in the progranulin gene. Brain 2006; 129:3081-3090.

-34 Josephs KA, Ahmed Z, Katsuse O, Parisi JF, Boeve BF, Knopman DS, Petersen RC, Davies P, Duara R, Graff-Radford NR, Uitti RJ, Rademakers R, Adamson J, Baker M, Hutton ML, Dickson DW: Neuropathologic features of frontotemporal lobar degeneration with ubiquitin-positive inclusions with progranulin gene (PGRN) mutations. J Neuropathol Exp Neurol 2007;66:142-151.

- 35 Schroder R, Watts GD, Mehta SG, Evert BO, Broich P, Fliessbach K, Pauls K, Hans VH, Kimonis V, Thal DR: Mutant valosin-containing protein causes a novel type of frontotemporal dementia. Ann Neurol 2005;57: 457-461.

- 36 Neumann M, Mackenzie IR, Cairns NJ, Boyer PJ, Markesbery WR, Smith CD, Taylor JP, Kretzschmar HA, Kimonis VE, Forman MS: TDP-43 in the ubiquitin pathology of frontotemporal dementia with VCP gene mutations. J Neuropathol Exp Neurol 2007;66: 152-157. 
\$37 Skibinski G, Parkinson NJ, Brown JM, Chakrabarti L, Lloyd SL, Hummerich H, Nielsen JE, Hodges JR, Spillantini MG, Thusgaard T, Brandner S, Brun A, Rossor MN, Gade A, Johannsen P, Sorensen SA, Gydesen S, Fisher EM, Collinge J: Mutations in the endosomal ESCRTIII-complex subunit CHMP2B in frontotemporal dementia. Nat Genet 2005;37:806-808.

- 38 Holm IE: Ubiquitin-positive inclusions in frontotemporal dementia linked to chromosome 3 (FTD-3). Brain Pathol 2006;16:S43.

-39 Mishra M, Paunesku T, Woloschak GE, Siddique T, Zhu LJ, Lin S, Greco K, Bigio EH: Gene expression analysis of frontotemporal lobar degeneration of the motor neuron disease type with ubiquitinated inclusions. Acta Neuropathol (Berl) 2007;114:81-94.

40 Schumacher A, Friedrich P, Diehl-Schmid J, Ibach B, Perneczky R, Eisele T, Vukovich R, Foerstl H, Riemenschneider M: No association of TDP-43 with sporadic frontotemporal dementia. Neurobiol Aging 2007, E-pub ahead of print.

-41 Rollinson S, Snowden JS, Neary D, Morrison KE, Mann DM, Pickering-Brown SM: TDP43 gene analysis in frontotemporal lobar degeneration. Neurosci Lett 2007;419:1-4.

42 Pasinelli P, Brown RH: Molecular biology of amyotrophic lateral sclerosis: insights from genetics. Nat Rev Neurosci 2006;7:710-723.

-43 Gros-Louis F, Gaspar C, Rouleau GA: Genetics of familial and sporadic amyotrophic lateral sclerosis. Biochim Biophys Acta 2006; 1762:956-972.

- 44 Morita M, Al Chalabi A, Andersen PM, Hosler B, Sapp P, Englund E, Mitchell JE, Habgood JJ, de Belleroche J, Xi J, Jongjaroenprasert W, Horvitz HR, Gunnarsson LG, Brown RH Jr: A locus on chromosome 9p confers susceptibility to ALS and frontotemporal dementia. Neurology 2006;66:839844.
45 Talbot K, Ansorge O: Recent advances in the genetics of amyotrophic lateral sclerosis and frontotemporal dementia: common pathways in neurodegenerative disease. Hum Mol Genet 2006;15:R182-R187.

46 Leigh PN, Whitwell H, Garofalo O, Buller J, Swash M, Martin JE, Gallo JM, Weller RO, Anderton BH: Ubiquitin-immunoreactive intraneuronal inclusions in amyotrophic lateral sclerosis. Morphology, distribution, specificity. Brain 1991;114:775-788.

47 Mackenzie IR, Bigio EH, Ince PG, Geser F, Neumann M, Cairns NJ, Kwong LK, Forman MS, Ravits J, Stewart H, Eisen A, McClusky L, Kretzschmar HA, Monoranu CM, Highley JR, Kirby J, Siddique T, Shaw PJ, Lee VM, Trojanowski JQ: Pathological TDP-43 distinguishes sporadic amyotrophic lateral sclerosis from amyotrophic lateral sclerosis with SOD1 mutations. Ann Neurol 2007;61: 427-434.

48 Tan CF, Eguchi H, Tagawa A, Onodera O, Iwasaki T, Tsujino A, Nishizawa M, Kakita A, Takahashi H: TDP-43 immunoreactivity in neuronal inclusions in familial amyotrophic lateral sclerosis with or without SOD1 gene mutation. Acta Neuropathol (Berl) 2007;113:535-542.

49 Mackenzie IR, Feldman H: The relationship between extramotor ubiquitin-immunoreactive neuronal inclusions and dementia in motor neuron disease. Acta Neuropathol (Berl) 2003;105:98-102.

50 Dickson DW, Josephs KA, Amador-Ortiz C: TDP-43 in differential diagnosis of motor neuron disorders. Acta Neuropathol (Berl) 2007;114:71-79.

51 Robertson J, Sanelli T, Xiao S, Yang W, Horne P, Hammond R, Pioro EP, Strong MJ: Lack of TDP-43 abnormalities in mutant SOD1 transgenic mice shows disparity with ALS. Neurosci Lett 2007;420:128-132.

-52 Hasegawa M, Arai T, Akiyama H, Nonaka T, Mori H, Hashimoto T, Yamazaki M, Oyanagi K: TDP-43 is deposited in the Guam parkinsonism-dementia complex brains. Brain 2007;130:1386-1394.
53 Leverenz JB, Yu CE, Montine TJ, Steinbart E, Bekris LM, Zabetian C, Kwong LK, Lee VM, Schellenberg GD, Bird TD: A novel progranulin mutation associated with variable clinical presentation and tau, TDP43 and $\alpha$-synuclein pathology. Brain 2007;130:1360-1374.

54 Amador-Ortiz C, Lin WL, Ahmed Z, Personett D, Davies P, Duara R, Graff-Radford NR, Hutton ML, Dickson DW: TDP-43 immunoreactivity in hippocampal sclerosis and Alzheimer's disease. Ann Neurol 2007; 61:435-445.

55 Nakashima-Yasuda H, Uryu K, Robinson J, Xie SX, Hurtig H, Duda JE, Arnold SE, Siderowf A, Grossman M, Leverenz JB, Woltjer R, Lopez OL, Hamilton R, Tsuang DW, Galasko D, Masliah E, Kaye J, Clark CM, Montine TJ, Lee VM, Trojanowski JQ: Comorbidity of TDP-43 proteinopathy in Lewy body related diseases. Acta Neuropathol (Berl) 2007;114:221-229.

56 Geser F, Winton MJ, Kwong LK, Yan X, Xie SX, Igaz LM, Garruto R, Perl D, Galasko D, Lee VM, Trojanowski JQ: Pathological TDP43 in parkinsonism-dementia complex and amyotrophic lateral sclerosis of Guam. Acta Neuropathol (Berl) 2007 DOI: 10.1007/ s00401-007-0257-y.

57 Kwong LK, Neumann M, Sampathu DM, Lee VM, Trojanowski JQ: TDP-43 proteinopathy: the neuropathology underlying major forms of sporadic and familial frontotemporal lobar degeneration and motor neuron disease. Acta Neuropathol (Berl) 2007;114: 63-70.

58 Neumann M, Kwong LK, Sampathu DM, Trojanowski JQ, Lee VM: TDP-43 proteinopathy in frontotemporal lobar degeneration and amyotrophic lateral sclerosis: Protein misfolding diseases without amyloidosis. Arch Neurol 2007;64:1388-1394.

59 Neumann M: TDP-43 proteinopathies: a new class of proteinopathies. Future Neurol 2007 , in press. 